\title{
You've Got Photos! The design and evaluation of a location-based media-sharing application
}

\author{
Niko Kotilainen \\ Department of Mathematical Information \\ Technology \\ University of Jyväskylä \\ Finland
}

\author{
Maria Papadopouli \\ Department of Computer Science \\ University of Crete \& \\ Institute of Computer Science \\ Foundation for Research \& Technology-Hellas
}

\begin{abstract}
PhotoJournal is a novel location-based media sharing application that enables users to build interactive journals that associate multimedia files with locations on maps and share this information with other users. Its underlying information discovery and sharing mechanism is 7DS that runs in either pure peer-to-peer or centralized server-to-client mode, depending on the availability of a server and/or an infrastructure. 7DS-enabled devices act as miniature caches, sharing information with each other. When access to an information server (e.g., web server) is not available, the local 7DS instance running on the device enables the device to search and access information from other peers in proximity. We have implemented the prototype and evaluated the delay to access the data using three testbeds. Two of these testbeds employ a centralized (server-to-client) architecture, while the third one applies the peer-to-peer paradigm. Depending on the underlying network technology and device capabilities, this delay varies. The results encourage us to perform additional empirical-based studies under increased traffic load conditions and initiate a user-study in the premises of a museum and a research park.
\end{abstract}

\section{INTRODUCTION}

New applications and tools for sharing and experimenting with multimedia data in a synchronous or asynchronous manner, such as Flickr, YouTube, Me.dium, MySpace, facebook, and JumpCut, have enriched on-line collaboration, allowing the formation of new types of social networks, interactions, and online communities. Furthermore, the market of location-based services grows rapidly. In the near future, mobile devices that have the processing, communication,

\footnotetext{
* This research was supported by the Greek General Secretariat for Research and Technology under Regional of Crete,

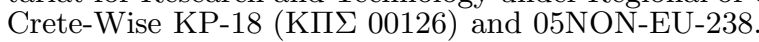

and geolocating capabilities will enable seamless integration of services combining media sharing and geographical tagging.

PhotoJournal applies the peer-to-peer (p2p) paradigm to facilitate the access and sharing of location-based multimedia content among mobile devices. It also enables users to build interactive multimedia "journals" that associate multimedia objects, such as pictures, video, or hypertext, with locations on maps. Multimedia files can be "superimposed" on certain locations of maps and users may manage, review, update or delete them. Users may query for content regarding an area of a map and update their journal. Peers that run an instance of the PhotoJournal application on a device may respond to such queries and share their multimedia content.

PhotoJournal is supported by graphical user interfaces (GUIs) to access, search, share, and manage the multimedia content and a middleware with two main components, namely a positioning and an information discovery and sharing system. The underlying positioning technologies are GPS and the Cooperative Location-sensing System (CLS) [9, 22], while 7DS [20] enables information discovery and sharing. When access to an information server (e.g., web server) is not available (e.g., a device experiences intermittent connectivity to the Internet), the 7DS instance running on that device (e.g., peer) enables the peer to search and access information from other peers in the wireless LAN. 7DS can instantiate both the server-to-client and peer-to-peer paradigms and provide complementary access through peers, when an infrastructure - or connectivity to an infrastructure - is not available. 7DS assumes that, in the face of disconnections, users can trade the data consistency and currency over data availability.

We implemented PhotoJournal and evaluated its performance under both peer-to-peer and infrastructure-based architectures. The delay that a user experiences to access the requested data from the time the device is in the range of another cooperative device with relevant data (i.e., dataholder) is measured. Depending on the underlying network technology (e.g., 3G or IEEE802.11), architecture, and device capabilities, the median delay varies from $282 \mathrm{~ms}$ (in a p2p architecture, running on a PC in a IEEE802.11 single-hop network) to $1.9 \mathrm{~s}$ (running the application on a smartphone and accessing the web server via a $3 \mathrm{G}$ network). However, the frequency that a device is close to a dataholder depends on several parameters, such as popularity of the data, den-
Mobimedia 2008 July 7-9, 2008, Oulu, Finland.

Copyright 2008 ICST ISBN 978-963-9799-25-7/08/07

DOI 10.4108/ICST.MOBIMEDIA2008. 4034 
sity of peers, mobility pattern, and transmission power.

Section 2 presents the related work, while Section 3 focuses on the PhotoJournal architecture and introduces its main components. The performance of the PhotoJournal is analyzed in Section 4. Finally, Section 5 reflects on mobile peer-to-peer computing and Section 6 summarizes our main conclusions and future work plans.

\section{RELATED WORK}

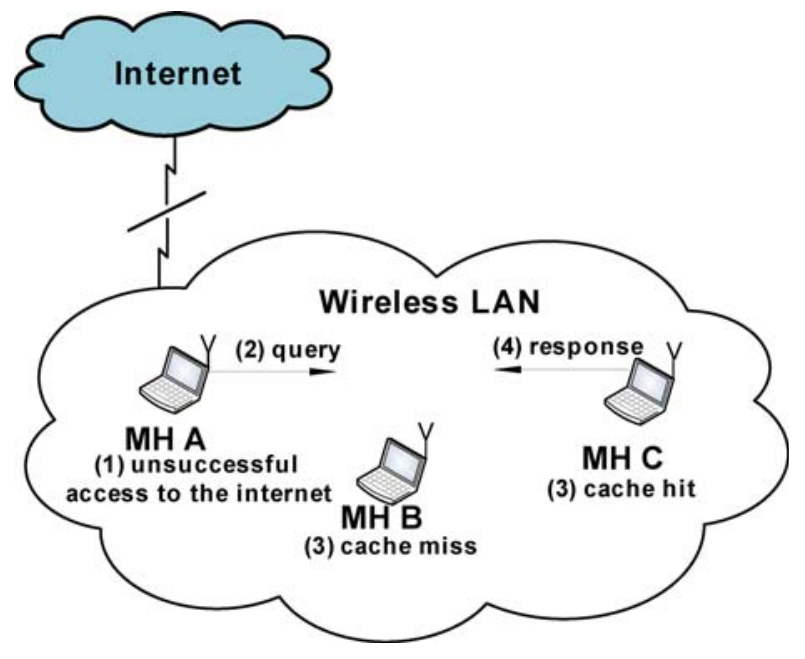

Figure 1: Example of information sharing using 7DS. The arrows show the message exchange for the 7DS communication. The light-shaded area denotes the wireless LAN, the darker-shaded area the Internet, and the thunderbolt-like shape the WAN connection that is not currently available.

The anticipation of a growing number of users that form "on-line" communities to gossip, share information and resources via their wireless-enabled devices inspired the design of 7DS. 7DS may relay, search for and disseminate information in a self-organizing manner, without the need for an infrastructure. 7DS-enabled devices can interact either in a $\mathrm{p} 2 \mathrm{p}$ or server-to-client manner. These different modes of operation allow 7DS to instantiate different mobile information access schemes when possible, and provide complementary access through peers, when an infrastructure is not available. Figure 1 illustrates an example of 7DS peer-to-peer use. Mobile host A (MH A) tries to access a data object. The local 7DS instance running on host A detects an unsuccessful attempt to connect to the Internet and tries to retrieve the data from peers that are within its wireless range. Both hosts $\mathrm{B}$ and $\mathrm{C}$ (MHB and $\mathrm{MHC}$, respectively) are within the range of host $\mathrm{A}$ and receive the query. Unlike host $\mathrm{B}$, host $\mathrm{C}$ has a copy of the data in its cache and responds to host A's query.

Applications interact with 7DS employing pairs of attributes to describe the data that they are willing to share with other application instances running on peers. For each application, 7DS maintains an index of the local cache that is populated with data that can be shared. This data may have been acquired from other peers or servers [18, 19, 20, 21].

MOBY [11] proposes a service-oriented network architecture, in which each peer interacts both with the available infrastructure and its neighbours. It provides a method to integrate available services in handheld mobile devices. MOBY's architecture is based on Mnode super-peers, which allow mobile devices to access and locate available services, as opposed to the 7DS platform, where there is no need for external storage. Although super-peers are mainly responsible for service management, interaction among Mnodes is encouraged in order to reduce load on peers acting as gateways. Horozov et al. in [11] discuss security challenges by integrating secure service registration capabilities in the available architecture.

Mobile chedar [15] is a middleware extension to Chedar [4], providing resource sharing and distribution in mobile p2p systems, in a completely decentralized fashion. The proposed API performs topology management by selecting connections that aim to establish a scalable and fault-tolerant network. Communication among peers in both Chedar and mobile Chedar is Gnutella-like, in which queries are sent to neighboring peers and direct connections are created among them. P2P systems that adopt the Chedar API are not evaluated in terms of performance, and no analysis is provided concerning the impact of malicious users on system security.

LightPeers [7] is a lightweight mobile p2p platform, developed to support users utilizing a variety of mobile devices with limited capabilities. Communication among peers is established by broadcasting discovery messages and multicasting queries to nodes of the same group. LightPeers was implemented to ease the exchange of information and services among peers and support interactive applications. This architecture can be used in ad-hoc networks that facilitate delay tolerant messaging.

Proem is a Java oriented middleware platform for developing and deploying applications for mobile ad-hoc networks. The Proem middleware consists of three parts: an application runtime environment, a set of middleware services, and a protocol stack for communication. In Proem, each application is managed by the peerlet engine, which is responsible for dynamically adding and removing peerlets from the system. The set of middleware functions is designed to allow distributed applications to share resources and exchange event information, and declare and discover new services. The protocol stack defines the syntax and semantics required to enable communication between peers.

This paper considers that in the wireless range of a querier, there is a cooperative device with the relevant data (in the $\mathrm{p} 2 \mathrm{p}$ architecture) or a predefined web server that can be accessed via the wireless Internet (in the infrastructure architecture), respectively. The frequency that a device is in the range of dataholders has a dominant impact on the total delay that a user will experience, i.e., the total time elapsed from the formation of the query until the local device receives relevant data. However, this delay depends on several parameters, such as popularity of the data, density of peers, their mobility pattern and transmission power. An evaluation of the impact of these parameters on data dissemination assuming random-walk based mobility patterns can be found in $[18,19,20,21]$. In general, for different usage and application characteristics, the likelihood that users in proximity would be interested in similar data varies.

Although currently 7DS uses single-hop multicast, a routing protocol could facilitate the communication among peers. Mobile peer-to-peer computing applications often create sparse and intermittently-connected networks, referred to as de- 
lay tolerant networks (DTNs). Traditional routing protocols for ad-hoc networks do not perform well in DTNs due to their unstable paths. Important parameters in the design of such routing protocols are the co-residency time between peers, time that a peer is out of the range of other relay nodes, information servers, or access points (APs), relaying, querying, and cooperation policies, information locality, and buffer management. Several studies on routing protocols in DTNs have appeared, evaluating the impact of buffer management, relaying policies, and placement of relay nodes (e.g., $[8,5,17,25])$ or of the knowledge about device location, peer movement and connectivity patterns on the routing protocol (e.g., [16, 13, 23, 24]). Depending on the communication patterns between peers, different network topologies can be formed, affecting dramatically the speed that the information is disseminated. Analyzing how fast data spread in scale-free networks has been the focus of recent studies (e.g., [14]).

\section{PHOTOJOURNAL ARCHITECTURE}

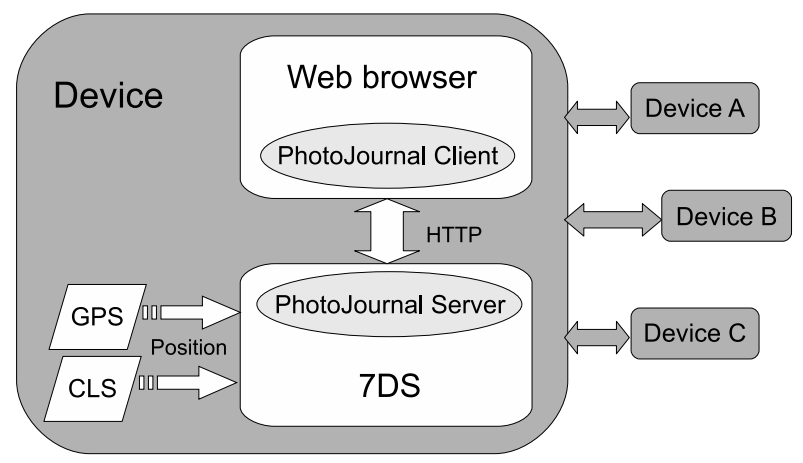

Figure 2: PhotoJournal architecture: The application is supported by an underlying information discovery and sharing mechanisms (7DS) and positioning system (e.g., CLS, GPS).

PhotoJournal allows the creation of interactive locationbased multimedia journals and enables users to discover and share their content with each other. A local PhotoJournal instance may automatically superimpose local multimedia content to the appropriate areas of maps and enable a user to specify location-based queries for certain areas of a map. It is supported by 7DS, its underlying information sharing and discovery mechanism, and a positioning system, GPS and CLS or outdoor and indoor environments, respectively. PhotoJournal runs as an application with a web browserbased user frontend.

\subsection{CLS}

CLS applies the peer-to-peer paradigm by enabling devices to gather positioning information from other neighboring peers, estimate their distance from their peers based on signal-strength measurements, and position themselves accordingly [9]. CLS creates a signal-strength signature map of the physical space during a training phase and compares it with analogous run-time measurements employing various statistical-based criteria. Iteratively, it can refine its positioning estimates by incorporating newly received information from other devices.
CLS and GPS periodically record the coordinates of the current position of the device with a timestamp in the positioning trace. Users can upload pictures and videos with their associated timestamp. PhotoJournal can correlate the timestamp information of the multimedia content with the positioning trace and associate the multimedia files with certain areas of a map. ${ }^{1}$

\subsection{User frontend}

The user frontend of the PhotoJournal client is a webbrowser based interface that communicates with the local PhotoJournal server using HTTP. The latter is responsible for the interaction of the PhotoJournal client with the 7DS module, forwarding the corresponding messages and assuring that packets are in the proper format as they are exchanged across different modules.

Using a PhotoJournal GUI, a user may superimpose multimedia content on certain locations of the map by clicking on the map and browsing the multimedia files corresponding to that location. Moreover, a user can add, modify, or delete comments about a certain multimedia file, rate its content and set its access permission. A multimedia file can be set public or private - only public files can be shared with other peers. The PhotoJournal frontend (as shown in Figure 4) runs on a web browser and consists of a map frame on the right and a photo bar on the left side of the window. Its backend runs on 7DS. It receives all queries from the frontend through 7DS's proxy server, and supports the typical 7DS functionality by adding or deleting photos, querying photos from 7DS neighbors or handing photos from the local cache. 7DS can also cache map files, enabling the application to work without an Internet connection.

PhotoJournal can automatically superimpose the uploaded content on an appropriate map by matching the timestamp of the content of the multimedia files with the timestamp of the GPS/CLS trace and associating these files with the corresponding position on the map. Furthermore, it updates its local 7DS cache and its indexing mechanism.

Figure 2 summarizes the main components of the locationbased media sharing system, namely the PhotoJournal application, 7DS and CLS.

\subsection{Peer discovery and information access}

A user may search for multimedia content related to a certain location in the following manner: First, the user indicates the region of interest by marking the corresponding area on the displayed map (e.g., the white rectangular on the map illustrated in Figure 4). Then, the local 7DS instance will search for relevant data in its cache, on the web, and in the cache of other peers. Specifically, it will first check its local cache for multimedia files associated with that area. If the search is successful, it will display a marker with a number indicating the number of multimedia files associated with that location. In the case that no relevant data can be found, 7DS's web client attempts to acquire it from the Internet by accessing a predefined web site. Finally, if the web client fails to acquire the requested data (e.g., in the case of intermittent connectivity to the Internet or unavailability of a web server), 7DS will form a media query and multicast it to its peers. A media query describes the requested data

${ }^{1}$ We assumed that the digital camera timestamps recorded files and is synchronized with the user's device running a positioning system. 


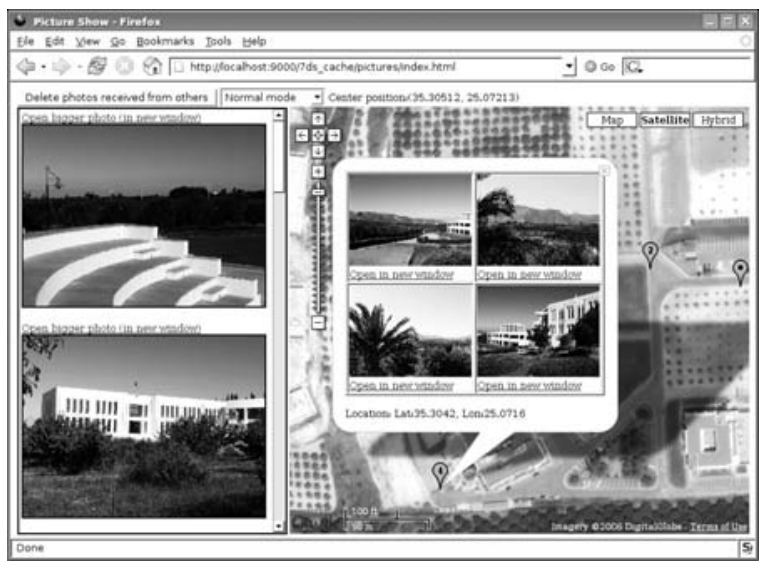

Figure 3: PhotoJournal can superimpose multimedia objects at their locations on a map. A marker indicates the number of files associated with that location.

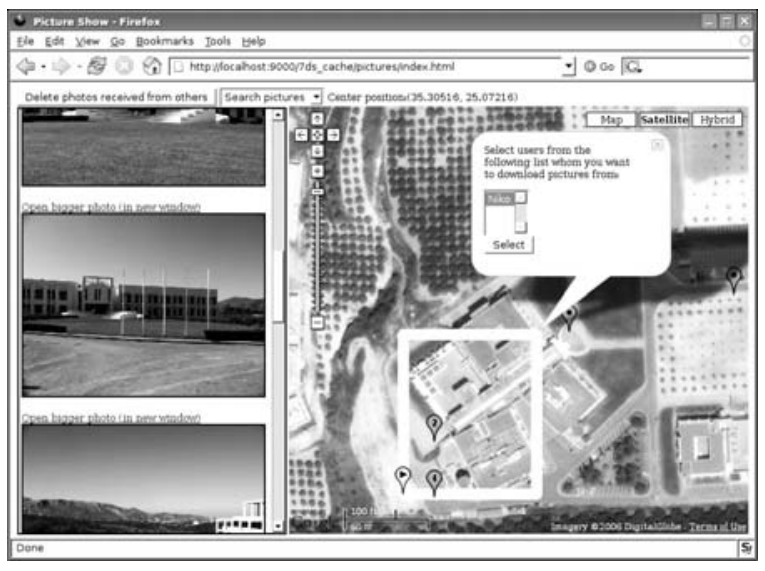

Figure 4: On the main GUI of the PhotoJournal a user can mark the area for which multimedia information will be requested.

in XML format and is formed using location- and rate-based criteria. Upon the reception of a media query, the local 7DS instance of a peer may search its local cache for relevant data. If a relevant data object is found in its cache, it will form and send an XML response, including a URL to the relevant multimedia file, reviews and rating information.

The PhotoJournal will display - periodically updating itthe list of peers that responded to recent queries. A user may select a certain 7DS peer from this list to retrieve the requested content. When the web client retrieves the relevant data objects from the peer, it stores them in the local cache and displays them on the map (as illustrated in Figures 3 and 4). Areas on the map associated with multimedia files can be distinguished by a marker that also indicates the number of the available relevant files. A demo of the PhotoJournal can be found in [2].

\section{PERFORMANCE ANALYSIS}

To evaluate the performance of the PhotoJournal, empiricalbased measurements were performed using two different ar- chitectures, namely, an ad-hoc and an infrastructure-based one. The infrastructure-based approach realizes the traditional server-to-client approach: PhotoJournal clients request content from a web server. Unlike the infrastructurebased architecture, in the ad-hoc (i.e., p2p) architecture, devices access the information in a peer-to-peer manner. In the infrastructure-based architecture, we experimented with both $3 \mathrm{G}$ or IEEE802.11 technologies. The estimated effective downlink speed of the $3 \mathrm{G}$ connection is approximately $400 \mathrm{Kbps}$. The peers in the $\mathrm{p} 2 \mathrm{p}$ architecture communicate via IEEE802.11 in the ad hoc mode.

Throughout the text, the terms "infrastructure" and "centralized" are used interchangeably for describing the architecture paradigm and testbed (similarly with the terms "adhoc" and "p2p").

\subsection{Metrics}

To evaluate the performance of the PhotoJournal application over the two different architectures, the following benchmarks are defined:

1. Query processing delay: the total time elapsed between the reception of a (neighbor or a media) query and the transmission of a response.

2. Query transmission delay: the time spent by a specific query to travel over the network, subject only to network elements and propagation delays.

3. Query forming delay: the time that a request spends at various levels of the protocol stack before being forwarded to the network layer.

\subsection{Testbeds}

The empirical-based measurements were performed on three testbeds. Two of them employed the infrastructure-based architecture, while the third one the ad-hoc based one. The client-to-server communication takes place using $3 \mathrm{G}$ (in the "3G infrastructure" testbed) and IEEE802.11 (in the "IEEE802.11 infrastructure" testbed). In these testbeds, the wireless client is a Nokia N80 smartphone and the web server runs Apache. In the ad-hoc testbed, wireless clients running the PhotoJournal application are part of an IEEE802.11b adhoc network, each using a PC equipped with an A-Link USB WLAN interface.

To measure these different delays, monitors are placed at certain testbed locations. The time granularity of these measurements depends on the specific platform. For example, the time-keeping clock of the smartphone has a frequency of $64 \mathrm{~Hz}$ (corresponding to a granularity of approximately $15 \mathrm{~ms}$ [10]), while the granularity of the web server monitor is of $1 \mu \mathrm{s}$. Table 1 illustrates the list of monitors used in our measurements along with the specific event they capture.

The main difference between the $p 2 p$ and the centralized architecture is that the latter does not require a peer (neighbor) discovery phase and querying devices send their requests directly to a predefined web server via either the $3 \mathrm{G}$ or IEEE802.11 infrastructure.

We ran 30 experiments using PhotoJournal in each of the three testbeds. In the infrastructure-based experiments, a script initiated a sequence of queries for the same content. For each experiment in the $\mathrm{p} 2 \mathrm{p}$ testbed, a user selected a different region of interest on the map, initiating a query for related content. The resulted delay measurements are shown in Figures 5, 6, and 7. 


\begin{tabular}{|c|l|}
\hline \multicolumn{2}{|c|}{ P2P architecture } \\
\hline Time & Event description \\
\hline$T_{1}$ & local 7DS instance receives a request \\
\hline$T_{2}$ & local 7DS multicasts a discovery query \\
\hline$T_{3}$ & peer receives a query \\
\hline$T_{4}$ & dataholder sends a response \\
\hline$T_{5}$ & querier receives response \\
\hline \multicolumn{2}{|c|}{ Centralized architecture } \\
\hline Time & Event description \\
\hline$T_{6}$ & smartphone receives a user query \\
\hline$T_{7}$ & smartphone sends a query to web server \\
\hline$T_{8}$ & web server receives a query \\
\hline$T_{9}$ & web server sends response to smartphone \\
\hline$T_{10}$ & smartphone receives response \\
\hline
\end{tabular}

Table 1: Monitors capturing various event types in the infrastructure and $\mathrm{p} 2 \mathrm{p}$ architectures. The term $T_{i}$ indicates the time the event $i$ was recorded at the corresponding monitor.

\begin{tabular}{|l|l|l|}
\hline Delay & P2P & Infrastructure \\
\hline Query forming & $T_{2}-T_{1}$ & $T_{7}-T_{6}$ \\
\hline Query processing & $T_{4}-T_{3}$ & $T_{9}-T_{8}$ \\
\hline Query transmitting & $T_{5}-T_{2}-T_{4}+T_{3}$ & $T_{10}-T_{7}-T_{9}+T_{8}$ \\
\hline
\end{tabular}

Table 2: Different delay types as measured based on the recorded event times for the p2p- and infrastructure-based architectures.

\subsection{Query forming delay}

The processing power and CPU load impact the query forming delay. In the infrastructure architecture, the query forming delay is significantly larger, exhibiting also higher variability compared to the ad-hoc one (as shown in Figure 5). The PhotoJournal query in the centralized testbed is formed by a smartphone with scarce resources and low processing capabilities compared to the powerful PCs used in the p2p testbed. Furthermore, compared to IEEE802.11 LANs, 3G networks are more demanding in terms of processing power. However, even when a PhotoJournal-client runs on a powerful PC, the query forming delay remains significantly large, up to $100 \mathrm{~ms}$. Such values are due to the XML document processing performed by 7DS in order to describe the requested items. Moreover, the interaction between the 7DS component and the PhotoJournal client results in frequent context switches, increasing further the measured delay.

As expected, query forming delay is larger in the case of media queries compared to neighbor queries, since in the former case the corresponding request needs to describe the area of interest whereas in the latter case, a peer discovery request is simply a "template" message (used to search for peers in the wireless range of the querier).

\subsection{Query processing delay}

The query processing delay is significantly lower in the centralized setting compared to the $\mathrm{p} 2 \mathrm{p}$ one (as shown in Figure 6). This is due to the complexity that 7Ds introduces, when used in networks where no 3G or IEEE802.11 infrastructure is available. The time required for a web server to form a response is significantly lower from the one of a regu-

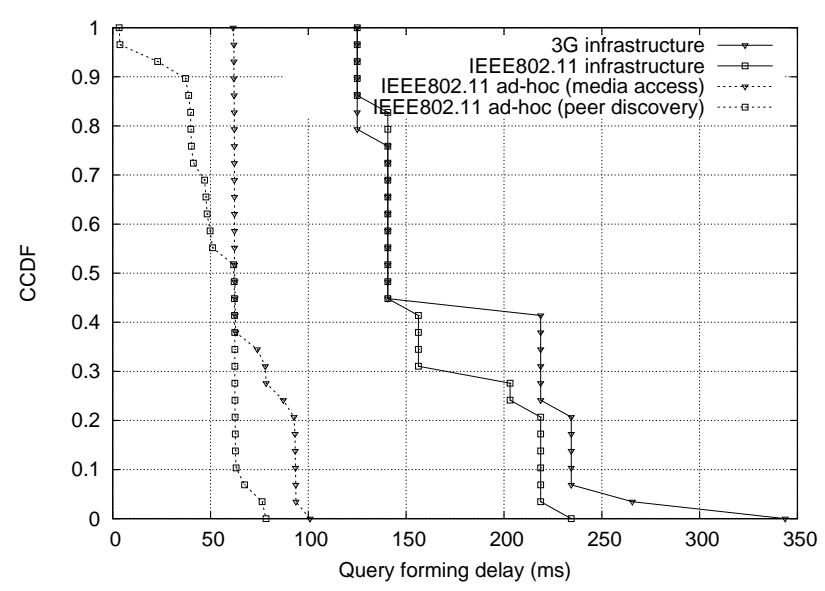

Figure 5: Complementary cumulative distribution function (CCDF) of query forming delays.

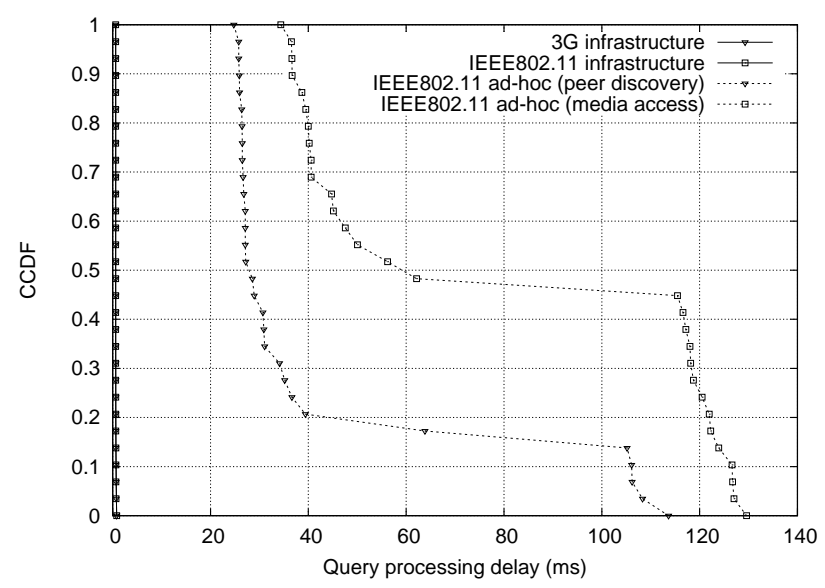

Figure 6: Complementary cumulative distribution function (CCDF) of query processing delays.

lar 7DS peer. Note that in the ad-hoc testbed, 7DS employs XML for describing a data item, while in the infrastructurebased architecture, a 7DS client only sends a simple HTTP request directly to the web server over the Internet. The total time elapsed between the reception of a media query and the transmission of a response is greater than $40 \mathrm{~ms}$ for the $70 \%$ of the queries in p2p mode (as illustrated in Figure 6).

The query processing delay for media access is larger than for peer discovery due to the intense processing required for the first query type. When a peer receives a media query, it will search the local cache for relevant media items. The variability exhibited in the ad-hoc architecture is due to the variable sized map areas.

\subsection{Query transmission delay}

The transmission delay of a media access query is stochastically larger than the delay of a peer discovery one (as shown in Figure 7). For example, in p2p, the median media access delay is approximately $39 \mathrm{~ms}$ compared to a median peer discovery delay of $15 \mathrm{~ms}$. A typical response size to a media access query is approximately $29 \mathrm{~KB}$ (compared to only a few bytes which is the response to a peer discovery one). 


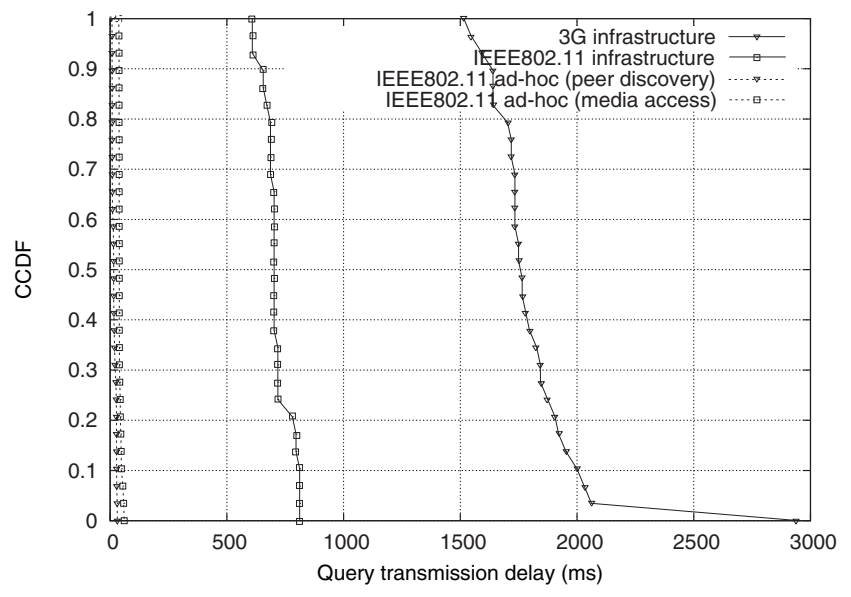

Figure 7: Complementary cumulative distribution function (CCDF) of query transmission delays.

The variability in query transmission delay is due to the various sizes of the response for different query types. Also compared to the ad-hoc network, an infrastructure results in increased transmission delays due to the aggregate traffic of other clients associated with the IEEE802.11 AP and the lower transfer rates (in 3G). The IEEE802.11 centralized approach exhibits lower transmission delays than $3 \mathrm{G}$ (e.g., median delay of $750 \mathrm{~ms}$ compared to $1800 \mathrm{~ms}$ ). With 3G's maximum speed of $2 \mathrm{Mbps}$, it is hard to compete with the 11 Mbps transfer rate of IEEE802.11.

To summarize, the mean and median delays from the time the PhotoJournal received user's input until the reception of the relevant media files are are $1,995 \mathrm{~ms}$ and $1,898 \mathrm{~ms}$ in $3 \mathrm{G}$, $876 \mathrm{~ms}$ and $843 \mathrm{~ms}$ in IEEE 802.11 infrastructure, and $305 \mathrm{~ms}$ and $282 \mathrm{~ms}$ in IEEE 802.11 ad-hoc, respectively. Thus, the 7DS/PhotoJournal introduces delays that are tolerable. However, several aspects of the current 7DS implementation can be improved; For instance, the code is large and complex. It can be simplified significantly using libraries included in recent Java versions. We intend to evaluate its scalability under increased traffic load conditions. Furthermore, it would be interesting to perform a user study in the premises of a museum or FORTH and collect additional feedback from users (e.g., visitors in these premises) not only about its performance but also its features and GUIs.

\section{DISCUSSION ON MOBILE P2P SYSTEMS}

The development of 7DS and PhotoJournal motivated us to reflect on mobile peer-to-peer computing. Critical aspects of a mobile peer-to-peer system are the incentives for cooperation and privacy requirements.

The effectiveness of mobile peer-to-peer computing systems depends on their substantial deployment, cooperation, interoperability, and scalability. Depending on the availability of a resource, a peer may dynamically adapt its cooperation strategy. The scarcity of resources enhances the tension between cooperation and competition. Given the energy constraints, the nondeterministic characteristics of the environment, and the presence of exogenous parameters that impact the resource availability, such resource allocation algorithms are non-trivial. In general, the following parameters impact the power consumption of a network interface: size and number of packets sent and received, and time the network interface is on. To reduce the power consumption, these parameters need to be kept low.

To prevent denial of service attacks, encourage cooperation, and better allocate resources, the use of micropaymentbased and/or reputation-based mechanisms can be important $[3,6,26,12,1]$. However, these mechanisms should have a relatively low overhead, in order to not discourage the energetic participation of peers. While a relaxed protection of resources may impede the use of a peer-to-peer system, high costs or strict conditions to access the resources may dissuade their usage. The design of a mobile $\mathrm{p} 2 \mathrm{p}$ system needs to address the balance between these two requirements.

Increasingly wireless devices collect a large amount of information that can be analyzed to reveal the personal and social context of the user. This abundance of information makes users vulnerable to intrusion of privacy threats. The identification of the position of the device and potentially, the identity of the subject using the device - which can be acquired directly or inferred using statistical analysis-are examples of such threats. Malicious users can abuse such information by spamming users with advertisements or disclosing it inappropriately. Thus, a tradeoff between enhancing the information access and disclosing private information inappropriately is exposed. The larger the availability of information, the more likely is to enhance the information access and sharing but also the higher the vulnerability in privacy threats.

As in the case of the Internet, peer-to-peer systems need to be flexible and dynamic to sustain long-term use. Privacy will play an important role in the adoption of mobile peer-to-peer computing applications. Currently, 7DS and PhotoJournal offer a crude distinction between private and non-private objects and a finer way to describe their privacy requirements is needed. However, privacy is context sensitive and depends on the social context, user activity, ownership of the device, application, and personality of the user. Depending on these parameters, the system may decide about the privacy and cooperation policies with or without any user intervention. Thus, it is important to provide mechanisms that allow a fine-level description of the privacy requirements and draw a balance between enhancing the service and protecting user privacy.

\section{CONCLUSIONS}

This work focused on PhotoJournal, a multimedia locationbased application, and analyzed the delay that the application experiences from the time the request is formed until a response is received. Depending on the underlying network technology and device capabilities, this median delay varies from $282 \mathrm{~ms}$ to $1,9 \mathrm{~s}$. In these experiments, in the wireless range of a querier, there was always a cooperative device with the relevant data (in the ad-hoc testbed) or a predefined web server that can be accessed via the wireless Internet (in the infrastructure testbeds), respectively. As mentioned earlier, the frequency that a device is in the range of dataholders has a great impact on the total delay that a user will experience, i.e., the total time elapsed from the formation of a query until the local device receives relevant data. Our earlier research analyzed the data dissemination in ad hoc wireless network, assuming random-walk based mobility models. An interesting followup study would consider 
heterogeneous wireless environments, supported partially by wireless infrastructures; in areas with limited or no coverage by APs, the mobile peer-to-peer computing paradigm can be used to enhance the information access. In such environments, it would be useful to evaluate various routing protocols integrated with mobile peer-to-peer systems using more realistic access and traffic patterns.

Only a few studies on mobile p2p systems evaluate the performance of their system with empirical-based measurements. Typically, the evolution of a technology includes the following steps: simulation-based studies of the technology, measurements in a real-life testbed and controlled experiments, and further empirical-based measurement studies in large-scale testbeds (if the technology becomes widely adopted). To assist the deployment of mobile peer-to-peer computing systems, a fruitful approach would include the development of the following components [21]:

- a general infrastructure for mobile peer-to-peer applications and a toolkit that new applications could use

- robust mobile peer-to-peer applications with friendly GUIs that can also control the distribution of data and form context- and semantic-based queries

- protocols that ensure anonymity and privacy

- mechanisms that encourage cooperation among peers in an energy-efficient manner

Mobile p2p computing opens up exciting challenges in computer science, demanding interdisciplinary research and innovative paradigms.

\section{ACKNOWLEDGEMENT}

The authors would like to thank Manolis Ploumidis and Elias Raftopoulos for their help in reviewing an earlier draft and providing useful feedback on the performance analysis and Alexandros Kapravelos, Despina Maggina, and Vasiliki Papavasileiou for their help in the implementation of some features of the PhotoJournal.

\section{REFERENCES}

[1] Ling liu. security and trust in peer-to-peer systems: Risks and countermeasures. http://www.cc.gatech.edu/ lingliu/keynotes/.

[2] Mobile Computing Activity at FORTH-ICS. http://www.ics.forth.gr/mobile/software.html/.

[3] Peer-to-peer: Harnessing the power of disruptive technologies.

http://www.freehaven.net/doc/oreilly/accountabilitych16.html.

[4] A. Auvinen, M. Vapa, M. Weber, N. Kotilainen, and J. Vuori. Chedar: Peer-to-peer middleware. In Proceedings of the 19th IEEE International Parallel \& Distributed Processing Symposium (IPDPS 2006), Rhodes Island, Greece, July 2006.

[5] Brendan Burns, Oliver Brock, and Brian Neil Levine. Autonomous Enhancement of Disruption Tolerant Networks. In Proc. IEEE International Conference on Robotics and Automation, Orlando, Florida, May 2006.

[6] Levente Buttyan and Jean-Pierre Hubaux. Nuglets: a virtual currency to stimulate cooperation in self-organized mobile ad hoc networks. Technical Report DSC/2001/001, Swiss Federal Institute of Technology, Lausanne, January 2001.
[7] Bent Guldbjerg Christensen. Lightpeers: A lightweight mobile p2p platform. In Proceedings of the Fifth IEEE International Conference on Pervasive Computing and Communications Workshops (PERCOMW'O7), pages 132-136, White Plains, NY, March 2007.

[8] James Davis, Andy Fagg, and Brian Neil Levine. Wearable computers as packet transport mechanisms in highly partitioned ad-hoc networks. In Proc. International Symposium on Wearable Computers (ISWC), Zurich, October 2001.

[9] Charalampos Fretzagias and Maria Papadopouli. Cooperative Location Sensing for Wireless Networks. In Second IEEE International conference on Pervasive Computing and Communications, Orlando, Florida, March 2004.

[10] Richard Harrison. Symbian $O S C++$ for Mobile Phones. John Wiley \& Sons Ltd, 2003.

[11] T. Horozov, A. Grama, V. Vasudevan, and S. Landis. Moby - a mobile peer-to-peer service and data network. In Proceedings of International Conference on Parallel Processing, pages 437-444, Washington, DC, USA, August 2002.

[12] Jean-Pierre Hubaux, Levente Butyan, and Srdan Capkun. The quest for security in mobile ad hoc networks. In $A C M$ International Symposium on Mobile Ad Hoc Networking and Computing (MobiHoc), pages 146-155, Long Beach, CA, October 2001

[13] Sushant Jain, Kevin Fall, and Rabin Patra. Routing in a delay-tolerant network. In ACM Symposium on Communications Architectures and Protocols (SigComm), Portland, OR, USA, August 2004.

[14] Jon Kleinberg. The wireless epidemic. Nature (News and Views), 449:287-288, 2007.

[15] Niko Kotilainen, Matthieu Weber, Mikko Vapa, and Jarkko Vuori. Mobile Chedar - a peer-to-peer middleware for mobile devices. In Proceedings of the Second International Workshop on Mobile Peer-to-Peer Computing (MP2P'05), pages 86-90, Kauai Island, Hawaii, March 2005.

[16] Qun Li and Daniela Rus. Sending messages to mobile users in disconnected ad-hoc wireless networks. In $A C M$ International Conference on Mobile Computing and Networking (MobiCom), pages 44-55, Boston, MA, USA August 2000

[17] Marc Liberatore, Brian Neil Levine, and Chadi Barakat. Maximizing Transfer Opportunities in Bluetooth DTNs. In Proc. ACM Conference on Future Networking Technologies (CoNext), Lisboa, Portugal, December 2006.

[18] Maria Papadopouli and Henning Schulzrinne. Seven degrees of separation in mobile ad hoc networks. In IEEE Conference on Global Communications (GLOBECOM), San Francisco, CA, November 2000.

[19] Maria Papadopouli and Henning Schulzrinne. Effects of power conservation, wireless coverage and cooperation on data dissemination among mobile devices. In $A C M$ International Symposium on Mobile Ad Hoc Networking and Computing (Mobihoc), Long Beach, CA, October 2001.

[20] Maria Papadopouli and Henning Schulzrinne. A performance analysis of 7DS a peer-to-peer data dissemination and prefetching tool for mobile users. In Advances in wired and wireless communications, IEEE Sarnoff Symposium Digest, Ewing, NJ, March 2001.

[21] Maria Papadopouli and Henning Schulzrinne. Peer-to-Peer Computing for Mobile Networks: Information Discovery and Dissemination. Springer (under preparation), 2008.

[22] Konstantinos Vandikas, Lito Kriara, Tonia Papakonstantinou, Anastasia Katranidou, Haris Baltzakis, and Maria Papadopouli. Empirical-based analysis of a cooperative location-sensing system. In ACM First International Conference on Autonomic Computing and Communication Systems (Autonomics), Rome, Italy, October 2007.

[23] J. Yang, C.-K. Lee Y. Chen, and M. Ammar. Ferry 
replacement protocols in sparse manet message ferrying

systems. In IEEE Wireless Communications and

Networking (WCNC), New Orleans, LA, March 2005.

[24] Wenrui Zhao, Mostafa Ammar, and Ellen Zegura. A message ferrying approach for data delivery in sparse mobile ad hoc networks. In IEEE Conference on Computer Communications (InfoCom), Hong Kong, March 2004.

[25] Wenrui Zhao, Yang Chen, Mostafa Ammar, Mark D.

Corner, Brian Neil Levine, and Ellen Zegura. Capacity Enhancement using Throwboxes in DTNs. In IEEE International Conference on Mobile Ad hoc and Sensor Systems, Vancouver, Canada, October 2006.

[26] Lidong Zhou and Zygmunt J. Haas. Securing ad hoc networks. IEEE Network, 13(6), November 1999 